\title{
Indicators of Workplace Burnout Among Physicians, Advanced Practice Clinicians, and Staff in Small to Medium-Sized Primary Care Practices
}

\author{
Debora Goetz Goldberg, PhD, MHA, MBA, Tulay G. Soylu, PhD, MHA, MBA, \\ Victoria M. Grady, PhD, Panagiota Kitsantas, PhD, James D. Grady, DMD, and \\ Len M. Nichols, PhD
}

Background: The rising prevalence of burnout among physicians and other healthcare professionals has become a major concern in the United States. Identifying indicators of burnout could help reduce negative consequences such as turnover, loss of productivity, and adverse health behaviors. The goal of this study was to examine whether individual behaviors and attitudes towards major disruptive change has an effect on workplace burnout.

Methods: This study analyzed survey responses from 1273 healthcare professionals from 154 small to medium-sized primary care practices participating in the EvidenceNOW initiative in Virginia. Healthcare professionals' behaviors and attitudes, such as anxiety and withdrawal, were assessed to determine associations with workplace burnout. Results were examined by professional role.

Results: Workplace burnout was reported by $31.6 \%$ of the physicians, $17.2 \%$ of advanced practice clinicians, $18.9 \%$ of clinical support staff, and $17.5 \%$ of administrative staff. Regardless of burnout status, results show all healthcare professional groups had high levels of anxiety. Providers had significantly higher scores for anxiety than all other healthcare professionals. Providers who experienced higher levels of anxiety and withdrawal were more than three times as likely to report burnout compared to those who experienced low levels in these domains.

Conclusions: Understanding individual behaviors and attitudes towards disruptive change may help practice leaders and policymakers develop strategies to reduce burnout among healthcare professionals. Programs should focus on strengthening the work environment of small to medium-sized practices to improve organizational capacity for change and address high levels of anxiety experienced by physicians, advanced practice clinicians and staff. (J Am Board Fam Med 2020;33:378-385.)

Keywords: Capacity Building, Health Personnel, Personnel Turnover, Physicians, Primary Health Care, Professional Burnout, Professional Role, Surveys and Questionnaires, Virginia, Workplace

This article was externally peer reviewed.

Submitted 23 July 2019; revised 11 October 2019; accepted 14 October 2019.

From George Mason University, Department of Health Administration and Policy, Fairfax, VA (DG, TGS, PK); George Mason University, School of Business, Fairfax, VA (VMG); Pivot Point Business Solutions (JDG); George Mason University, Center for Health Policy Research and Ethics, Fairfax, VA (LMN).

Funding: This research was funded by the Agency for Health Care Research and Quality (R18HS023913).

Conflicting and Competing Interests: D. Goldberg, T. Soylu, P. Kitsantas, and L. Nichols report no conflict of interest. V. Grady and J. Grady report proprietary interest in the Change Diagnostic Index $\bigodot$ instrument.

Corresponding author: Debora Goetz Goldberg, PhD, MHA, MBA, George Mason University, Department of Health Administration and Policy, Peterson Family Hall, MS IJ3, 4400 University Drive Fairfax, VA 22030 (E-mail: dgoldbe4@gmu.edu)

\section{Introduction}

The rising prevalence of burnout among physicians and other health care professionals has become a major policy concern in the United States. ${ }^{1}$ Burnout is defined as a "syndrome of emotional exhaustion, depersonalization, and a sense of low personal accomplishment that results in decreased effectiveness at work." ${ }^{2}$ Numerous studies have concluded that burnout is a serious problem for physicians, advanced practice clinicians, nurses, and other health care professionals. ${ }^{3-10}$ Recent research indicates that primary care physicians experience a higher rate of burnout than most other physician specialties. ${ }^{11}$ There are limited studies that compare the level of burnout between primary care 
physicians and other types of health care professionals. One recent study found that job satisfaction was slightly higher among nurse practitioners than physicians in primary care clinics. ${ }^{12}$

The leading causes for burnout among physicians in the United States include bureaucratic tasks, physicians' loss of autonomy, long hours on computerized work, ongoing tasks related to quality improvement and payer requirements, ${ }^{7,9,13}$ implementation of electronic health records (EHRs), and misalignment of values between providers and organizational leaders. ${ }^{14-16}$ Burnout can have a significant negative impact on the quality of patient care by negatively influencing clinical decision making, increasing medical errors and malpractice claims, and lowering patient satisfaction. ${ }^{17-19}$ Burnout may also lead to high turnover, difficult relationships between providers and staff, and drug and alcohol abuse..$^{20,21}$

In addition, a recent study by Han et $\mathrm{al}^{22}$ found that physician turnover and reduced clinical hours attributable to burnout result in approximately $\$ 4.6$ billion in costs each year in the United States.

Primary care physicians, advanced practice clinicians, and staff experience a tremendous number of workplace changes brought about by the adoption and use of EHRs and other information technology, transformation to new care delivery models such as a patient-centered medical home or accountable care organization, transfer of practice ownership, and/or compliance to numerous regulatory and payer requirements. Research indicates that small to medium-sized practices, defined as those with 1 to 15 providers, experience more challenges during major disruptive change efforts that may have an influence on workplace burnout. Among these challenges are a lack of resources, time scarcity, physician shortage, and high turnover rates. $^{23,24}$

Considering that burnout may lead to dissatisfaction, quality of care concerns, and provider and staff turnover, there is a strong need to understand behaviors and attitudes associated with burnout in primary care practices. ${ }^{25}$ Additional knowledge on indicators of burnout can be used to develop approaches to identify physicians, advanced practice clinicians, and practice staff that experience workplace dissatisfaction or exhaustion and to develop mechanisms to support these individuals. The goal of this study was to examine health care professionals' behaviors and attitudes to major disruptive changes in primary care practices in Virginia and to understand whether these reactions have an effect on workplace burnout.

\section{Methods}

This study analyzed cross-sectional survey data collected in 2018 by the Heart of Virginia Health care $(\mathrm{HVH})$ cooperative. $\mathrm{HVH}$ was 1 of 7 cooperatives in the United States funded by the Agency for Health care Research and Quality's EvidenceNOW initiative, ${ }^{26}$ which aimed to support quality improvement efforts in small to medium-sized primary care practices across the country. The intervention focused on implementation of the "ABCS" of Heart Disease and Stroke Prevention ${ }^{27}$ that includes aspirin use by high-risk individuals, blood pressure control, cholesterol management, and smoking cessation. The $\mathrm{HVH}$ initiative also assisted participating practices with activities to increase quality improvement, such as the use of advanced EHR functions for patient registries and data measurement and reporting.

The HVH cooperative recruited 203 small to medium-sized primary care practices in Virginia to participate in the 3 -year initiative. Of those practices, 154 fielded a practice member survey to providers and staff, which led to a $76 \%$ response rate of practices participating in this part of the HVH initiative. The resulting survey database consisted of 1273 primary care physicians, advanced practice clinicians (nurse practitioners and physician assistants), clinical support staff (eg, licensed practical nurses and medical assistants), and administrative staff (eg, office managers, receptionists, and medical billing professionals). These groups of health care professionals were developed due to small sample sizes in multiple health professional categories. Missing data for the analytic sample ranged from $0.3 \%$ to $0.9 \%$. The study was approved by the George Mason University Institutional Review Board in September 2017.

\section{Measures}

In this study, burnout was measured through a selfreported practice member survey instrument. Individuals were asked about their degree of burnout by using a nonproprietary single-item burnout indicator that instructs respondents to define burnout for themselves. The intent of the question was to capture the general state of well-being among physicians, advanced practice clinicians, and 
practice staff. This metric has been used in previous studies to measure burnout among physicians. ${ }^{28-30}$ Respondents were asked to choose 1 from 5 possible answers: (1) "I enjoy my work. I have no symptoms of burnout," (2) "Occasionally I am under stress, and I do not always have as much energy as I once did, but I do not feel burned out," (3) "I am definitely burning out and have 1 or more symptoms of burnout, such as physical and emotional exhaustion," (4) "The symptoms of burnout that I am experiencing will not go away. I think about frustrations at work a lot," and (5) "I feel completely burned out and often wonder if I can go on practicing. I am at the point where I may need some changes." If the participants' responses on the above burnout question were 3 or higher, they were classified as being "burned out."

Measuring individual response to change is important because individual employees that have difficulty transitioning may exhibit viewpoints and behaviors that lead to withdrawal, depression, or low morale. Employee reactions to change may also have a collective influence on organizational effectiveness. The Change Diagnostic Index@ (CDI) examines feelings, emotions, and attitudes of individuals as a response to organizational and technological change. ${ }^{31,32}$ The CDI has been used in numerous industries to study employee reactions to major change efforts, including primary care and specialty physician practices, outpatient clinics, and hospitals. The CDI was used in this study to measure behaviors and attitudes of providers and staff in response to participation in the $\mathrm{HVH}$ initiative, which required practices to implement a clinical intervention along with numerous practice transformation activities.

The CDI instrument consists of 25 questions based on a 5-point scale (strongly agree to strongly disagree) that measure employees' perceptions and behaviors in 7 domains: anxiety, frustration, delayed development, rejection of the environment, refusal to participate, withdrawal, and global. The instrument identifies an individual's level of anxiety, which is characterized by signs of tension, distress, and apprehension. Frustration is identified through agitation and when an individual has difficulty completing tasks. Rejection of the environment is identified through protest, anger, and whether the individual views the change as forced. Delayed development identifies whether an individual has ceased to progress in their professional role, which could result from a perceived threat to competence, relatedness, or autonomy. Refusal to participate identifies whether an individual denies an aspect of the change or has a decrease in their level of work presence and job contribution. Withdrawal identifies personal detachment, defined as physical and/ or physiologic distance between the employee and the organization. The global domain is a collective measure of employees' reaction to organizational change. Previous research identified elevated scores in CDI domain areas associated with a loss of effectiveness on work tasks and decreased overall job performance. Reliability analyses of the CDI scale in this study, and in previously published research, ${ }^{32}$ resulted in a Cronbach's $\alpha$ score above 0.7 for all CDI domains, indicating internal consistency of the scale.

Other measures included in the analysis are the individual respondent's number of hours worked per week and practice-level characteristics that consider practice size, single- or multispecialty practice, and whether a practice is located in an underserved area. Practice size was categorized into the following groups: 1 to 5 clinicians, 6 to 10 clinicians, and 11 or more clinicians.

\section{Statistical Approach}

Descriptive statistics and $\mathrm{c}^{2}$ analysis were conducted to assess the distribution of burnout status in this sample and to determine whether there was a significant association between burnout status and type of health care professional. One-way analysis of variance with Bonferroni correction for multiple comparisons were used to examine significant differences in the average score for each CDI domain among the various health care professional groups. Logistic regression was used to assess the effects of each CDI domain on burnout, controlling for practice size, single- or multispecialty practice, whether a practice is located in an underserved area, and an individual respondent's number of hours worked per week. Furthermore, separate logistic regression models were built for each type of health care professional group to examine differences in the relationship between CDI domains and burnout. Logistic regression models were adjusted for potential clustered data.

\section{Results}

The sample consisted of 1273 health care professionals, of which $11 \%$ were classified as physicians, 
Table 1. Distribution of Health Care Professional Groups and Burnout $(n=1273)$

\begin{tabular}{|c|c|c|c|c|c|}
\hline \multirow[b]{2}{*}{ Parameter } & \multirow[b]{2}{*}{ Sample, n (\%) } & \multicolumn{4}{|c|}{ Burnout } \\
\hline & & No, n (\%) & Yes, n (\%) & $P$ Value* & OR $(95 \% \mathrm{CI})^{\dagger}$ Burnout \\
\hline Burnout total & & $1024(80.1)$ & $249(19.5)$ & & \\
\hline Health Care Professionals & & & & 0.002 & \\
\hline Physicians & $139(11.0)$ & $93(68.4)$ & $43(31.6)$ & & $1.75(1.09-2.82)$ \\
\hline Advanced practice clinicians ${ }^{\ddagger}$ & $99(7.8)$ & $82(82.8)$ & $17(17.2)$ & & $1.01(0.54-1.88)$ \\
\hline Clinical support staff ${ }^{\S}$ & $451(35.6)$ & $365(81.1)$ & $85(18.9)$ & & $1.29(0.87-1.91)$ \\
\hline Administrative staff ${ }^{\|}$ & $578(45.6)$ & $477(82.5)$ & $101(17.5)$ & & Reference \\
\hline
\end{tabular}

*The $P$ value is based on $\chi^{2}$ analysis testing the association between burnout and the type of health care professional.

${ }^{\dagger}$ OR stands for odds ratios calculated from a logistic regression model that has been adjusted for practice size, single- or multispecialty practice, whether a practice is located in an underserved area, and number of hours worked per week

${ }^{\ddagger}$ Advanced practice clinicians include nurse practitioners and physician assistants.

${ }^{\S}$ Clinical support staff includes licensed practical nurses and medical assistants.

"Administrative staff includes office managers, receptionists, and medical billing professionals.

Bold text indicates statistically significant finding.

OR, odds ratio; CI, confidence interval.

$7.8 \%$ as advanced practice clinicians, $35.6 \%$ clinical support staff, and $45.6 \%$ administrative staff. In this sample, $19.5 \%$ of the health care professionals experienced burnout. Table 1 displays information on health care professional groups and burnout among health care professionals. A significant association was found between burnout and health care professional groups $(P<0.05)$. Specifically, $31.6 \%$ of the physicians, $17.2 \%$ of advanced practice clinicians, $18.9 \%$ of clinical support staff, and $17.5 \%$ of administrative staff reported burnout. Overall, physicians were 1.75 (95\% CI, 1.09-2.82) times more likely to report burnout than administrative staff after adjusting for the number of hours worked per week and practice characteristics.

Differences in the average scores of the CDI domains across the various health care professionals are reported in Table 2. On average, primary care physicians had a significantly $(P<0.001)$ higher score for anxiety than advanced practice clinicians, clinical support staff, and administrative staff. No other significant differences in the average scores of the CDI domains across the 4 health professional groups were observed. Regardless of burnout status, results show all health care professional groups had high levels of anxiety.

Table 2. Differences in the Change Diagnostic Index Domains by Health Care Professional Role at the Practice $(\mathrm{n}=\mathbf{1 2 7 3})$

\begin{tabular}{|c|c|c|c|c|c|c|}
\hline $\begin{array}{l}\text { Domains of the Change } \\
\text { Diagnostic Index }\end{array}$ & $\begin{array}{c}\text { Sample } \\
\text { Mean (SD) }\end{array}$ & $\begin{array}{l}\text { Physicians } \\
\text { Mean (SD) }\end{array}$ & $\begin{array}{c}\text { Advanced Practice } \\
\text { Clinicians* } \\
\text { Mean (SD) }\end{array}$ & $\begin{array}{c}\text { Clinical Support } \\
\text { Staff } \\
\text { Mean (SD) }\end{array}$ & $\begin{array}{c}\text { Administrative Staff } \\
\text { Mean (SD) }\end{array}$ & $P$ Value \\
\hline Global & $2.19(0.75)$ & $2.29(0.82)$ & $2.21(0.71)$ & $2.19(0.76)$ & $2.16(0.73)$ & 0.332 \\
\hline Anxiety & $2.50(0.84)$ & $2.77(0.88)$ & $2.57(0.83)$ & $2.46(0.83)$ & $2.44(0.83)$ & 0.000 \\
\hline Frustration & $1.83(0.71)$ & $1.76(0.71)$ & $1.80(0.73)$ & $1.85(0.72)$ & $1.83(0.70)$ & 0.627 \\
\hline Delayed development & $2.00(0.72)$ & $1.98(0.69)$ & $1.98(0.66)$ & $2.02(0.72)$ & $1.99(0.73)$ & 0.911 \\
\hline Rejection of environment & $1.97(0.75)$ & $2.01(0.78)$ & $2.04(0.71)$ & $1.99(0.77)$ & $1.94(0.74)$ & 0.438 \\
\hline Refusal & $1.74(0.67)$ & $1.66(0.64)$ & $1.72(0.63)$ & $1.78(0.71)$ & $1.72(0.64)$ & 0.266 \\
\hline Withdrawal & $1.70(0.70)$ & $1.60(0.76)$ & $1.69(0.66)$ & $1.73(0.72)$ & $1.71(0.68)$ & 0.353 \\
\hline
\end{tabular}

*Advanced practice clinicians include nurse practitioners and physician assistants.

${ }^{\dagger}$ Clinical support staff includes licensed practical nurses and medical assistants.

${ }^{\ddagger}$ Administrative staff includes office managers, receptionists, and medical billing professionals.

Bold text indicates statistically significant finding.

$\mathrm{SD}$, standard deviation. 
Table 3. Logistic Regression for Burnout among Health Care Professionals Based on the Change Diagnostic Index $(\mathrm{n}=1273)$

\begin{tabular}{|c|c|c|c|c|c|}
\hline $\begin{array}{l}\text { The Change Diagnostic } \\
\text { Index }\end{array}$ & $\begin{array}{c}\text { Entire Sample } \\
\text { Burnout, } \\
\text { OR }(95 \% \text { CI)* }\end{array}$ & $\begin{array}{c}\text { Physicians } \\
\text { Burnout, } \\
\text { OR }(95 \% \mathrm{CI})^{*}\end{array}$ & $\begin{array}{l}\text { Advanced Practice } \\
\text { Clinicians }{ }^{\dagger} \text { Burnout, } \\
\text { OR }(95 \% \text { CI) }\end{array}$ & $\begin{array}{l}\text { Clinical Support } \\
\text { Staff }{ }^{\ddagger} \text { Burnout, } \\
\text { OR }(95 \% \text { CI })^{*}\end{array}$ & $\begin{array}{c}\text { Administrative Staff } \\
\text { Burnout, } \\
\text { OR }(95 \% \mathrm{CI})^{*}\end{array}$ \\
\hline Global & $0.83(0.55-1.23)$ & $1.19(0.43-3.34)$ & $0.22(0.02-2.24)$ & $0.76(0.37-1.55)$ & $0.90(0.49-1.69)$ \\
\hline Anxiety & $2.26(1.67-3.07)$ & $3.22(1.42-7.31)$ & $7.97(1.74-36.5)$ & $1.65(1.02-2.73)$ & $2.31(1.39-3.85)$ \\
\hline Frustration & $1.64(1.05-2.56)$ & $3.46(0.65-18.4)$ & $4.25(0.07-245.1)$ & $1.32(0.72-2.42)$ & $2.04(0.89-4.65)$ \\
\hline Delayed development & $2.03(1.34-3.08)$ & $0.65(0.22-1.96)$ & $8.45(0.22-326.7)$ & $3.89(1.79-8.49)$ & $2.02(1.01-4.02)$ \\
\hline Rejection of environment & $1.85(1.16-2.93)$ & $1.93(0.75-4.93)$ & $57.2(1.56-2109.2)$ & $1.69(0.89-3.21)$ & $1.33(0.57-3.07)$ \\
\hline Refusal & $0.76(0.47-1.23)$ & $0.61(0.12-3.07)$ & $0.01(0.0004-3.41)$ & $0.82(0.44-1.53)$ & $0.83(0.39-1.71)$ \\
\hline Withdrawal & $1.09(0.75-1.59)$ & $4.15(1.18-14.5)$ & $3.52(0.38-32.3)$ & $0.78(0.39-1.59)$ & $0.97(0.51-1.87)$ \\
\hline
\end{tabular}

*Adjusted for practice size, single- or multispecialty practice, whether a practice is located in an underserved area, and number of hours worked per week.

${ }^{\dagger}$ Advanced practice clinicians include nurse practitioners and physician assistants.

${ }^{\ddagger}$ Clinical support staff includes licensed practical nurses and medical assistants.

${ }^{\S}$ Administrative staff includes office managers, receptionists, and medical billing professionals. Bold text indicates a statistically significant finding.

$\mathrm{OR}$, odds ratio; CI, confidence interval.

Overall, health care professionals were twice as likely to report burnout with higher levels of anxiety (odds ratio [OR]. 2.26; 95\% CI, 1.67-3.07) and frustration (OR, 1.64; 95\% CI, 1.05-2.56) than their counterparts with lower levels of anxiety and frustration, as shown in Table 3. They were also more likely to report burnout if they indicated higher levels of delayed development (OR, 2.03; 95\% CI, 1.34-3.08) and rejection of environment (OR, 1.85; 95\% CI, 1.16-2.93). When analyses were stratified by health care profession, we found that physicians who experienced increasing anxiety and withdrawal were more than 3 times as likely to report burnout compared with those who did not experience high levels of these domains. A significant association between increasing levels of anxiety and burnout was also found in advanced practice clinicians. In addition, increasing levels of anxiety and delayed development significantly raised the odds of burnout reporting among the administrative staff and clinical support staff.

\section{Discussion}

Our study identified a statistically significant difference in burnout rates among health professional groups in primary care practices. Of interest are the higher rates of burnout in primary care physicians than in other health professionals, such as nurses, medical assistants, and administrative staff. The findings of higher physician burnout than that in other professions are in line with previous research. ${ }^{6}$ These higher rates of burnout could be reflective of the greater accountability among physicians for patient care, practice management, and compliance to numerous external requirements from payers and regulators.

Our study also found elevated CDI scores among study participants experiencing burnout, specifically higher levels of anxiety, delayed development, and withdrawal. Anxiety was particularly high, especially for physicians experiencing burnout. High anxiety could indicate "psychological strain" 33 and possibly lead to lower morale (enthusiasm, confidence, or loyalty) of an individual or health care professional group. An increased level of anxiety could lead to decreases in work productivity and, if left untreated, could lead to mental health disorders or poor physical health. Elevated levels in the withdrawal domain among physicians reporting burnout could be an indicator of workplace dissatisfaction and feelings of helplessness. Withdrawal may lead to irritability, social isolation, and decreased work performance. Our study also found higher levels in the delayed development domain among clinical support staff and administrative staff reporting burnout, suggesting a loss of interest in personal development among these health professionals that could lead to decreased job-related motivation and performance. Previous research using the CDI indicates that elevated 
scores in these domains may lead to escalation of the refusal to participate domain, which could result in low work productivity, high absenteeism, or turnover. $^{32}$

A significant correlation between CDI domains and burnout suggests a possibility of addressing adverse perceptions and behaviors earlier to reduce turnover, increase physician and staff morale, and prevent or reduce the progression of burnout. Although we found that physicians experience higher burnout than other health care professionals, high anxiety levels were reported across health professional groups, indicating a need for programs and services that focus on all employees. Previous research on primary care practices' adaptive reserve, which measures the internal capacity for organizational learning and development, found higher rates of adaptive reserve associated with lower levels of burnout. $^{34}$ Our study reinforces the conclusions drawn from this earlier research and supports strengthening the work environment of small to medium-sized practices to improve the organizational capacity for change and reduction of burnout. Strategies to improve the work environment during organizational and technological change include increased support from leadership, targeted education and training, strong communication, and individual coaching. For example, previous research in primary care suggests that an increased focus on teamwork and face-to-face communication may improve job satisfaction and reduce burnout. ${ }^{12,35}$ Specific programs or services should also be developed to address the level of anxiety experienced by health care professionals during major transformations. Considering the lack of capacity and expertise in small and independent primary care practices, external assistance from local medical societies and associations may be needed.

Our study has several limitations that should be considered. Study data were collected from physicians, advanced practice clinicians, and staff at primary care practices in Virginia and may not be generalized to other regions. Practices who volunteered for the HVH study may exhibit characteristics different from the general population of practices in Virginia, which may result in a selection bias. It is possible that individuals in practices that agreed to participate in the $\mathrm{HVH}$ initiative were high performers or highly motivated and/or less prone to burnout. Data were obtained from a selfreport survey, which may produce response bias based on social desirability, extreme or midpoint response style, or other response behaviors that may affect measurement quality. In addition, the CDI was designed to study employee reaction to a single organizational or technological change, such as the adoption of EHRs or transfer of organizational ownership. However, the current primary care practice environment involves major changes to organizational structures and functions arising from multiple sources, which makes it difficult to study employee responses to a specific change effort. We acknowledge results from the CDI incorporate employee perceptions and behaviors on numerous practice changes that include participation in the HVH project as well as other major transformations.

\section{Conclusions}

Workplace burnout in primary care is a critical issue that was induced, in part, by major transformations to care delivery and administrative processes. It is critical to understand the dynamic factors associated with workplace burnout in primary care, which could help in the development of prevention and intervention strategies to reduce burnout, address workplace dissatisfaction, and prevent future turnover among physicians and other health care professionals. Policy makers should consider influences on health care professionals when developing new programs and regulatory requirements. Practice leaders may need external support to strengthen the work environment and to monitor the level and source of anxiety, withdrawal, conflict, and loss of interest experienced by physicians and other health care professionals during major transformations.

To see this article online, please go to: http://jabfm.org/content/ 33/3/378.full.

\section{References}

1. American Academy of Family Physicians (AAFP). Family physician burnout, well-being, and professional satisfaction. Available from: https://www. aafp.org/about/policies/all/physician-burnout.html. Accessed September 26, 2018.

2. Shanafelt TD, Sloan JA, Habermann TM. The wellbeing of physicians. Am J Med 2003;114:513-9.

3. Miller KI, Zook EG, Beth HE. Occupational differences in the influence of communication on stress and burnout in the workplace. Manag Commun Q 1989;3:166-90. 
4. Hernandez W, Yanchus NJ, Osatuke K. Evolving the JD-R model: the moderating effects of job resources and burnout taxonomies. Organ Dev J 2018;36:31-53.

5. Munnangi S, Dupiton L, Boutin A, Angus L. Burnout, perceived stress, and job satisfaction among trauma nurses at a level I safety-net trauma center. J Trauma Nurs 2018;25:4-13.

6. Shanafelt TD, Boone S, Tan L, et al. Burnout and satisfaction with work-life balance among US physicians relative to the general US population. Arch Intern Med 2012;172:1377-85.

7. Peckham C. Physician lifestyles-linking to burnout: a Medscape survey. Available from: https:// www.medscape.com/features/slideshow/lifestyle/ 2013/public\#6. Published 2013. Accessed January 31, 2019.

8. Shanafelt TD, Noseworthy JH. Executive leadership and physician well-being: Nine organizational strategies to promote engagement and reduce burnout. Mayo Clin Proc 2017;92:129-46.

9. Peckham C. Medscape lifestyle report 2017: race and ethnicity, bias and burnout. Available from: https://www.medscape.com/features/slideshow/ lifestyle/2017/overview. Published January 11, 2017. Accessed January 31, 2019.

10. Shanafelt TD, Hasan O, Lotte ND, et al. Changes in burnout and satisfaction with work-life balance in physician and the general US working population between 2011 and 2014. Mayo Clin Proc 2015;90: 1600-13.

11. Peckham C. Medscape National Physician Burnout \& Depression Report 2018. Available from: https:// www.medscape.com/slideshow/2018-lifestyle-burnoutdepression-6009235?faf=1\#1. Published January 17, 2018. Accessed January 31, 2019.

12. Mundt MP, Zakletskaia LI. Professional communication networks and job satisfaction in primary care clinics. Ann Fam Med 2019;17:428-35.

13. Leiter MP, Frank E, Matheson TJ. Demands, values, and burnout: relevance for physicians. Can Fam Physician 2009;5 5:1224-5.

14. Linzer M, Levine R, Meltzer D, Poplau S, Warde C, West CP. 10 bold steps to prevent burnout in general internal medicine. J Gen Intern Med 2014;29:18-20.

15. Noseworthy J, Madara J, Cosgrove D, et al. Physician burnout is a public health crisis: a message to our fellow health care CEOs. Available from: https://www.healthaffairs.org/do/10.1377/ hblog20170328.059397/full/. Published 2017. Accessed January 31, 2019.

16. The Physicians Foundation. 2018 Survey of America's physicians. Available from: https:// physiciansfoundation.org/wp-content/uploads/2018/ 09/physicians-survey-results-final-2018.pdf. Published 2018. Accessed January 31, 2019.
17. Linzer M, Manwell LB, Williams ES, et al. Working conditions in primary care: physician reactions and care quality. Ann Intern Med 2009;151:28-36.

18. Shanafelt TD, Balch CM, Bechamps G, et al. Burnout and medical errors among American surgeons. Ann Surg 2010;251:995-1000.

19. Kumar S. Burnout and doctors: prevalence, prevention and intervention. Healthcare (Basel) 2016;4:37.

20. Nash D. Physician burnout: what can be done? Available from: https://www.medpagetoday.com/ publichealthpolicy/generalprofessionalissues/71204. Published February 16, 2018. Accessed January 31, 2019.

21. Jabbarpour Y, Gold K, Chang D, Puffer J. Moving from burnout to wellness: identifying the problem and working towards solutions. Available from: https://www.graham-center.org/content/dam/rgc/ documents/press-events/primary-care-forum/ PrimaryCareBurnout.pdf. Published May 7, 2018. Accessed January 31, 2019.

22. Han S, Shanafelt TD, Sinsky CA, et al. Estimating the attributable cost of physician burnout in the United States. Ann Intern Med 2019;170:784-90.

23. Meyers DS, Clancy CM. Primary care: too important to fail. Ann Intern Med 2009;150:272-3.

24. Goldberg DG, Mick SS, Kuzel AJ, Feng LB, Love LE. Why do some primary care practices engage in practice improvement efforts whereas others do not? Health Serv Res 2013;48:398-416.

25. Buck K, Williamson M, Ogbeide S, Norberg B. Family physician burnout and resilience: a crosssectional analysis. Fam Med 2019;51:657-63.

26. US Department of Health and Human Services, Agency for Healthcare Research and Quality (AHRQ). Evidence Now: advancing heart health in primary care. Available from: https://www.ahrq. gov/evidencenow/index. Accessed June 10, 2017.

27. Centers for Disease Control and Prevention (CDC). National Heart Disease \& Stroke Prevention Program. Strategies for states to address the "ABCS" of heart disease and stroke prevention. 2010. Available from: https://www.cdc.gov/dhdsp/ programs/spha/docs/abcs_guide.pdf. Published 2010. Accessed July 22, 2019.

28. Rohland BM, Kruse GR, Rohrer JE. Validation of a single-item measure of burnout against the Maslach Burnout Inventory among physicians. Stress Health 2004;20:75-9.

29. Linzer M, the Healthy Work Place Investigators, Sinsky CA, Poplau S, Brown R, Williams E. Joy in medical practice: clinician satisfaction in the healthy work place trial. Health Aff 2017;36:1808-14.

30. Cuellar A, Krist AH, Nichols LM, Kuzel AJ. Effect of practice ownership on work environment, learning culture, psychological safety, and burnout. Ann Fam Med 2018;16:S44-S51. 
31. Grady V, Grady J. The pivot point: success in organizational change. New York (NY): MorganJames Publishing; 2013.

32. Grady V, Grady J. The relationship of Bowlby's attachment theory to the persistent failure of organizational change initiatives. J Chang Manag 2013;13:206-22.

33. Bliese PD, Britt TW. Social support, group consensus and stressor-strain relationships: social context matters. J Organiz Behav 2001;22:425-36.
34. Blechter B, Jiang N, Cleland C, Berry C, Ogedegbe O, Shelley D. Correlates of burnout in small independent primary care practices in an urban setting. J Am Board Fam Med 2018;31: 529-36.

35. Alidina S, Rosenthal MB, Schneider EC, Singer SJ, Friedberg MW. Practice environments and job satisfaction in patient-centered medical homes. Ann Fam Med 2014;12:331-7. 